\title{
The Clinical Significance and Risk Factors of Colorectal Stricture in Ulcerative Colitis
}

\author{
Sung Chul Park ${ }^{1}$ and Yoon Tae Jeen ${ }^{2}$ \\ ${ }^{I}$ Division of Gastroenterology and Hepatology, Department of Internal Medicine, Kangwon National University School of Medicine, Chuncheon, \\ and ${ }^{2}$ Division of Gastroenterology and Hepatology, Department of Internal Medicine, Korea University Anam Hospital, Korea University \\ College of Medicine, Seoul, Korea
}

\begin{abstract}
See "Risk Factors of Colorectal Stricture Associated with Developing High-Grade Dysplasia or Cancer in Ulcerative Colitis: A Multicenter Long-term Follow-up Study" by Weimin Xu, et al. on page 601, Vol. 14, No. 5, 2020
\end{abstract}

The management of ulcerative colitis (UC) patients with colorectal stricture remains a difficult task in clinical practice. The incidence of colorectal stricture in UC patients is known to range from $1.5 \%$ to $11.2 \%$, with reports varying, and the risk of developing cancer in UC patients with colorectal stricture was reported to fall in the range of $0 \%$ to $33 \% .^{1-3}$ In a relatively recent study by Fumery et al., ${ }^{1}$ the reported incidence of lowgrade dysplasia, high-grade dysplasia, and cancer in UC patients who underwent surgery for stricture without dysplasia or cancer on preoperative endoscopic biopsy was 2.5\%, 2.5\%, and 5\%, respectively. In another study, dysplasia was observed in 11 (73\%) out of 15 UC patients with stricture. ${ }^{3}$ Thus, an appropriate and systematic approach is necessary regarding the presence of dysplasia or cancer in UC patients with colorectal stricture.

Although pathogenesis for colorectal stricture in UC patients has not clearly been identified yet, it has been suggested that stricture is due to fibrosis in the bowel wall, the probable cause of which has been reported to be inflammatory neutrophils that produce basic-fibroblast growth factor. ${ }^{2}$ Few studies have investigated the risk factors for dysplasia or cancer in UC with colorectal stricture. In a previous study, the factors related to the probability of malignancy in UC with colorectal stricture were long-term disease duration $>20$ years, proximal location of stricture to splenic flexure, and symptomatic large bowel obstruction. ${ }^{4}$ In the study by Fumery et al., ${ }^{1}$ the only factor related to dysplasia or cancer in inflammatory bowel disease patients with stricture in the multivariate analysis, was no active disease at the time of surgery and age at stricture diagnosis showed a relation only in univariate analysis.

With increasing duration of UC, the cumulative risk of colorectal cancer (CRC) keeps increasing. The risk factors for colitis-associated colorectal neoplasia are known to include concurrent primary sclerosing cholangitis (PSC), extensive colitis, active endoscopic or histologic inflammation, a family history of CRC $<50$ years of age, personal history of dysplasia, and colorectal stricture. ${ }^{5,6}$ Accordingly, a screening colonoscopy is recommended for patients with left-sided or extensive colitis over 8 years after symptom onset as the risk of developing CRC is increased during such time, and to patients with concurrent PSC regardless of disease duration. The interval between surveillance colonoscopies should be adjusted in consideration of the risk of dysplasia and the time taken for cancer development, while an annual colonoscopy is recommended for patients with high-risk factors. $^{5}$

In this issue of Gut and Liver, Xu et al. ${ }^{7}$ analyzed UC patients registered in a prospectively maintained Chinese database, using univariate and multivariate logistic regression analyses, to examine the risk factors for colorectal stricture associated with UC carcinogenesis over long-term disease duration. The analysis included 246 eligible patients in total, and the median follow-up time was 13.0 years (interquartile range, 9.0-16.0). The results showed 35 cases (14.2\%) of colorectal stricture, and compared to those without stricture, patients with stricture had worse clinical outcomes including lower remission rate, higher serious complications (bleeding, intestinal obstruction, or perforation), increased need for surgery, and more frequent malignant trans-

Correspondence to: Yoon Tae Jeen

Department of Internal Medicine, Korea University Anam Hospital, Korea University College of Medicine, 73 Inchon-ro, Seongbuk-gu, Seoul 02841, Korea

Tel: +82-2-920-6555, Fax: +82-2-953-1943, E-mail: ytjeen@korea.ac.kr pISSN 1976-2283 eISSN 2005-1212 https://doi.org/10.5009/gnl20237

(c) This is an Open Access article distributed under the terms of the Creative Commons Attribution Non-Commercial License (http://creativecommons.org/licenses/by-nc/4.0) which permits unrestricted non-commercial use, distribution, and reproduction in any medium, provided the original work is properly cited. 
formation such as dysplasia (5.7\%) or CRC (25.7\%). However, such results may be also related to poorly controlled disease activity and severe inflammation, so it seems challenging for this retrospective study to elucidate the causal relationship between these outcomes and colorectal stricture.

In the eligible UC patients, a significant association with high-grade dysplasia or CRC was found for colorectal stricture (odds ratio [OR], 9.350; 95\% confidence interval [CI], 2.842 to 30.762), inflammatory polyps (OR, 5.464; 95\% CI, 1.692 to 17.638), disease duration $\geq 10$ years (OR, 3.223; 95\% CI, 1.040 to 9.985), and age $\geq 40$ years at the time of diagnosis (OR, 8.499; 95\% CI, 1.903 to 37.956). Notably, colorectal stricture showed the most significant effect on malignant transformation among several risk factors compared to the previous study in which it showed a borderline effect. ${ }^{8}$ In addition, the risk factors contributing to the development of colorectal stricture included disease duration $\geq 5$ years (OR, 3.211; 95\% CI, 1.168 to 8.881), moderate anemia (hemoglobin <90 g/L) (OR, 3.373; 95\% CI, 1.472 to 7.731), and PSC (OR, 5.842; 95\% CI, 1.395 to 24.468). The patients with risk factors for colorectal stricture in this study are likely to represent the group of patients with severe inflammation and poorly controlled disease severity. An interesting point is that PSC was included as a risk factor for colorectal stricture. In UC accompanied by PSC, the incidence of CRC is known to be high though the inflammation in the colon is not severe. A recent study reported that, in UC patients with PSC, a notable elevation of serum soluble CD40 was observed, while the CD40/ CD40 ligand system was associated with inflammation in atherothrombosis, suggesting that PSC may contribute to colorectal stricture via such an immunological response; however, further studies are required to determine its relationships. ${ }^{9,10}$

In this study, colorectal stricture in UC had worse clinical outcomes and a high risk of malignant transformation, while longer disease duration, moderate anemia, and PSC were risk factors for development of colorectal stricture. Thus, earlier colonoscopy surveillance and active treatment for inflammation control are needed to prevent stricture formation and malignant transformation in these patients. Further studies should be conducted for an appropriate and individualized approach and treatment of UC according to the carcinogenic risk factors such as colorectal stricture.

\section{CONFLICTS OF INTEREST}

No potential conflict of interest relevant to this article was reported.

\section{ORCID}

Sung Chul Park https://orcid.org/0000-0003-3215-6838

Yoon Tae Jeen https://orcid.org/0000-0003-0220-3816

\section{REFERENCES}

1. Fumery M, Pineton de Chambrun G, Stefanescu C, et al. Detection of dysplasia or cancer in 3.5\% of patients with inflammatory bowel disease and colonic strictures. Clin Gastroenterol Hepatol 2015;13:1770-1775.

2. Yamagata M, Mikami T, Tsuruta T, et al. Submucosal fibrosis and basic-fibroblast growth factor-positive neutrophils correlate with colonic stenosis in cases of ulcerative colitis. Digestion 2011;84:12-21.

3. Lashner BA, Turner BC, Bostwick DG, Frank PH, Hanauer SB. Dysplasia and cancer complicating strictures in ulcerative colitis. Dig Dis Sci 1990;35:349-352.

4. Gumaste V, Sachar DB, Greenstein AJ. Benign and malignant colorectal strictures in ulcerative colitis. Gut 1992;33:938-941.

5. Magro F, Gionchetti P, Eliakim R, et al. Third European evidencebased consensus on diagnosis and management of ulcerative colitis. Part 1: definitions, diagnosis, extra-intestinal manifestations, pregnancy, cancer surveillance, surgery, and ileo-anal pouch disorders. J Crohns Colitis 2017;11:649-670.

6. Shergill AK, Farraye FA. Toward a consensus on endoscopic surveillance of patients with colonic inflammatory bowel disease. Gastrointest Endosc Clin N Am 2014;24:469-481.

7. Xu W, Ding W, Gu Y, Cui L, Zhong J, Du P. Risk factors of colorectal stricture associated with developing high-grade dysplasia or cancer in ulcerative colitis: a multicenter long-term followup study. Gut Liver 2020;14:601-610.

8. Rutter MD, Saunders BP, Wilkinson KH, et al. Cancer surveillance in longstanding ulcerative colitis: endoscopic appearances help predict cancer risk. Gut 2004;53:1813-1816.

9. Lampinen M, Vessby J, Fredricsson A, Wanders A, Rorsman F, Carlson M. High serum sCD40 and a distinct colonic T cell profile in ulcerative colitis associated with primary sclerosing cholangitis. J Crohns Colitis 2019;13:341-350.

10. Antoniades C, Bakogiannis C, Tousoulis D, Antonopoulos AS, Stefanadis C. The CD40/CD40 ligand system: linking inflammation with atherothrombosis. J Am Coll Cardiol 2009;54:669-677. 